\title{
Tunnelling of plane waves through a square barrier
}

\author{
J. Julve \\ IMAFF, Consejo Superior de Investigaciones Científicas, Serrano 113 bis, Madrid \\ 28006, Spain \\ E-mail: julve@imaff.cfmac.csic.es
}

F. J. de Urríes

Departamento de Física, Universidad de Alcalá de Henares, Alcalá de Henares (Madrid), Spain

E-mail: fernando.urries@uah.es

\begin{abstract}
The time evolution of plane waves in the presence of a 1-dimensional square quantum barrier is considered. Comparison is made between the cases of an infinite and a cut-off (shutter) initial plane wave. The difference is relevant when the results are applied to the analysis of the tunnelling regime. This work is focused on the analytical calculation of the time-evolved solution and highlights the contribution of the resonant (Gamow) states.
\end{abstract}

PACS numbers: 11.10.Ef, 11.10.Lm, 04.60 


\section{Introduction}

The study of the traversal of potential barriers by wave packets in the 1-dimensional case is relevant for the electron transport through barrier junctions, the physics of wave guides and light transmission through Photonic Band Gaps, etc. On more theoretical grounds, the resonances, transient excitations and the time of arrival of wave packets, with ensuing paradoxes of non-locality and super-luminal tunnelling, are further interesting aspects.

Relevant resonances are mostly expected in systems of two or more barriers separated by a gap. Actually they generally occur in any simple barrier, and the plain square barrier is most tractable, fully representative for many theoretical purposes and devoid of bound states or other unessential features.

On the other hand, practical calculations face important limitations and the effort of pushing the analytical calculation as far as possible is always rewarding. Significant progress has been accomplished [1] for a Gaussian packet impinging on a square barrier, where the Gaussian structure was exploited, and [2] for the shutter problem, where the contribution of the resonant (Gamow) states in a double square barrier was worked out.

In this work we consider the time evolution, in the presence of a square barrier of height $V$ in the interval $[0, L]$, of an initial plane wave with support on the whole 1-D space, aiming to work out the contribution of the resonant states. Our calculation extends the results of the shutter problem, where the initial plane wave occupied the space axis at one side of the barrier and was specially suited to study details of the propagation of the wave front.

The reason for doing this is twofold. First, the simpler problem of the shutter initial condition $\psi_{s}(x)=\theta(-x) \mathrm{e}^{\mathrm{i} k x}$ has been assumed in the literature to be meaningful also for the analysis of the transmission in the tunnelling regime when $k \ll k_{V} \equiv \sqrt{2 m V}$. Unfortunately such a cut-off initial wave function is highly non-monochromatic as its Fourier transform shows:

$$
\tilde{\psi}_{s}(p)=\frac{1}{\sqrt{2 \pi}} \int_{-\infty}^{\infty} \mathrm{d} x \mathrm{e}^{-\mathrm{i} p x} \psi_{s}(x)=\frac{1}{\mathrm{i}} \frac{1}{\sqrt{2 \pi}} \mathrm{PP} \frac{1}{\mathrm{k}-\mathrm{p}}+\sqrt{\frac{\pi}{2}} \delta(\mathrm{k}-\mathrm{p})
$$

The Principal Part distribution around $k$ is exceedingly wide so that the amplitude of the momentum $p=k_{V}$ skimming the barrier is $\propto\left(k-k_{V}\right)^{-1}$, a sizeable value (in modulus, actually it is $>k_{V}^{-1}$ ) even for very small incident $k$, with a slow decrease for $p>k_{V}$. Secondly, by considering infinite plane waves the way is paved for further uses, since superpositions of them build up any desired initial wave packet. In particular, the compact result obtained for the Gaussian wave packet [1] could be re-derived pinpointing the contribution of each single Fourier component.

In this work we follow the approach used by Peierls and García-Calderón [3], namely Laplace-transforming the time evolution equation into a second order linear differential equation (SOLDE) in one variable, expressing its solutions in terms of the Green function (GF) with resonant boundary conditions (RBCs) and then undoing the Laplace transform. 
The first step is done in Section 2, where a brief discussion is made of the use of the Green's method for the solution of SOLDEs when the boundary conditions (BCs) required for the GF and for the solution are different. The GF with RBCs features a simple structure of isolated (resonance) poles, in terms of which the ( $p$-dependent) Laplace-transformed wave function is worked out in Section 3. Then the explicit calculation of the inverse Laplace transform leading to the sought after $t$-dependent solution is carried out in Section 4.

As a check of this cumbersome analytical calculation, the $t=0$ (Section 5 ) and the $t=\infty$ (Section 6) limits of the time-dependent solution $\psi(x, t)$ are calculated: at $t=0$ one must recover the initial plane wave and at $t=\infty$ a suitable stationary solution must be reached. Then the conclusions are drawn in Section 7. Some notations and a number of calculations and technical details are deferred to the Appendices.

\section{The evolution equations}

We consider the 1-D time-dependent Schrödinger equation

$$
\left(\mathrm{i} \hbar \frac{\partial}{\partial t}-H\right) \psi(x, t)=0
$$

where the Hamiltonian $H=p^{2} / 2 m+V(x)$ corresponds to the square barrier potential $V(x)=\theta(x) \theta(L-x) V$ and the solution satisfies the initial condition $\psi(x, 0) \equiv \psi_{o}(x)=$ $\mathrm{e}^{\mathrm{i} k x}, k>0$.

The Laplace transform $\bar{\psi}(s)=\int_{o}^{\infty} \mathrm{d} t \mathrm{e}^{-s t} \psi(t)$ on the time variable, brings the parabolic partial derivative differential equation (1) to the simpler SOLDE

$$
L_{x} \bar{\psi}(x, p) \equiv\left[\frac{\partial^{2}}{\partial x^{2}}+p^{2}-\frac{2 m}{\hbar^{2}} V(x)\right] \bar{\psi}(x, p)=\mathrm{i} \alpha \mathrm{e}^{\mathrm{i} k x}
$$

where $\alpha \equiv 2 m / \hbar$ and $p^{2}=\mathrm{i} \alpha s$ (Appendix A). Besides the usual scattering in and out solutions, the time-independent Schrödinger homogeneous equation $L_{x} \bar{\psi}=0$ has the resonant solutions satisfying the homogeneous outgoing RBCs

$$
\left.\partial_{x} \bar{\psi}\right|_{x=0}=-\mathrm{i} p \bar{\psi}(0),\left.\partial_{x} \bar{\psi}\right|_{x=L}=\mathrm{i} p \bar{\psi}(L),
$$

which exist only for a denumerable set of isolated values $p_{n}$ of $p$ lying in the lower half complex plane (Appendix A).

A solution to the inhomogeneous equation (2) for $p^{2} \in R_{+}$can be explicitly written down for each region of $V(x)$ :

$$
\begin{array}{ll}
\bar{\psi}_{I}(x, p)=B \mathrm{e}^{-\mathrm{i} p x}+\frac{\mathrm{i} \alpha}{p^{2}-k^{2}} \mathrm{e}^{\mathrm{i} k x} & (x<0) \\
\bar{\psi}_{I I}(x, p)=M \mathrm{e}^{\mathrm{i} p^{\prime} x}+N \mathrm{e}^{-\mathrm{i} p^{\prime} x}+\frac{\mathrm{i} \alpha}{p^{\prime 2}-k^{2}} \mathrm{e}^{\mathrm{i} k x} & (0 \leq x \leq L) \\
\bar{\psi}_{I I I}(x, p)=A \mathrm{e}^{\mathrm{i} p x}+\frac{\mathrm{i} \alpha}{p^{2}-k^{2}} \mathrm{e}^{\mathrm{i} k x} & (x>L)
\end{array}
$$

where the amplitudes $A, B, M$ and $N$ are functions of $p$ completely determined by the matching conditions at $x=0$ and $x=L$ and have a common denominator of the form 
$D(p) \equiv \ominus^{2} \mathrm{e}^{\mathrm{i} p^{\prime} L}-\oplus^{2} \mathrm{e}^{-\mathrm{i} p^{\prime} L}$ (notation in Appendix A). Out of all the terms of the general homogeneous solution, the choice of $\mathrm{e}^{\mathrm{i} p x}($ for $x>L)$ or $\mathrm{e}^{-\mathrm{i} p x}($ for $x<0)$ is dictated by the behaviour of the solution for $x \rightarrow \pm \infty$, since a small positive imaginary part in $p$ is supposed when performing the inverse Laplace transform leading to $\psi(x, t), t>0$ (Appendix D).

However, this expression of the solution is not suited to perform the inverse Laplace transform back to $\psi(x, t)$ because of the nontrivial analytical form of the amplitudes above. Instead the GF approach lets us to express $\bar{\psi}(x, p)$ as a sum of isolated pole terms plus other simple terms easier to deal with. To this end, (2) together with the Green equation for $G\left(x, x^{\prime}, p\right)$ can be written in the form

$$
\begin{aligned}
& L_{x} \bar{\psi}(x, p)-\mathrm{i} \alpha \mathrm{e}^{\mathrm{i} k x}=0 \\
& L_{x} G\left(x, x^{\prime}, p\right)-\delta\left(x-x^{\prime}\right)=0,
\end{aligned}
$$

where $G\left(x, x^{\prime}, p\right)$ is required to obey RBCs, namely

$$
\left.\partial_{x} G\left(x, x^{\prime}, p\right)\right|_{x=0}=-\mathrm{i} p G\left(0, x^{\prime}, p\right),\left.\partial_{x} G\left(x, x^{\prime}, p\right)\right|_{x=L}=\mathrm{i} p G\left(L, x^{\prime}, p\right)
$$

It is expected that $G\left(x, x^{\prime}, p\right)=L_{x}^{-1}$ will have poles at $p=p_{n}$, where the homogeneous equation $L_{x} \bar{\psi}=0$ with RBCs has non-trivial solutions.

The equations (5) and (6) may conveniently be given the short-hand notation $\Psi=0$ and $\Gamma=0$ respectively. Then the Green method uses the integral equation $\int_{0}^{L} \mathrm{~d} x[\bar{\psi} \Gamma-G \Psi]=0$ to obtain $\bar{\psi}(x, p)$ in terms of $G\left(x, x^{\prime}, p\right)$. Only if $\bar{\psi}$ and $G$ obey the same homogeneous BCs, the surface terms in this integral cancel out and one obtains the familiar result $\bar{\psi}_{I I}(x, p)=\int_{0}^{L} \mathrm{~d} x^{\prime} \mathrm{i} \alpha G\left(x^{\prime}, x, p\right) \mathrm{e}^{\mathrm{i} k x^{\prime}}$.

However the BCs for $\bar{\psi}(x, p)$ (involving $\bar{\psi}$ and $\partial_{x} \bar{\psi}$ both at $x=0$ and at $x=L$ ) stemming from (4) are different from (3) and non-homogeneous. In that case one obtains a modified expression for the region $I I$, namely

$$
\begin{aligned}
\bar{\psi}_{I I}(x, p)= & \int_{0}^{L} \mathrm{~d} x^{\prime} \mathrm{i} \alpha G\left(x^{\prime}, x, p\right) \mathrm{e}^{\mathrm{i} k x^{\prime}} \\
& -\frac{\alpha}{p+k} G(L, x, p) \mathrm{e}^{\mathrm{i} k L}-\frac{\alpha}{p-k} G(0, x, p)
\end{aligned}
$$

For the external regions $I$ and $I I I$, the matching of $\bar{\psi}$ at $x=0$ and $x=L$ determine the coefficients $B$ and $A$ respectively, so that

$$
\begin{aligned}
\bar{\psi}_{I}(x, p)= & \mathrm{i} \alpha \mathrm{e}^{-\mathrm{i} p x} \int_{0}^{L} \mathrm{~d} x^{\prime} \mathrm{i} \alpha G\left(x^{\prime}, 0, p\right) \mathrm{e}^{\mathrm{i} k x^{\prime}} \\
& -\frac{\alpha}{p+k} G(L, 0, p) \mathrm{e}^{\mathrm{i} k L} \mathrm{e}^{-\mathrm{i} p x}-\frac{\alpha}{p-k} G(0,0, p) \mathrm{e}^{-\mathrm{i} p x} \\
& -\frac{\mathrm{i} \alpha}{p^{2}-k^{2}} \mathrm{e}^{-\mathrm{i} p x}+\frac{\mathrm{i} \alpha}{p^{2}-k^{2}} \mathrm{e}^{\mathrm{i} k x}
\end{aligned}
$$

and

$$
\begin{aligned}
\bar{\psi}_{I I I}(x, p)= & \mathrm{i} \alpha \mathrm{e}^{\mathrm{i} p(x-L)} \int_{0}^{L} \mathrm{~d} x^{\prime} \mathrm{i} \alpha G\left(x^{\prime}, L, p\right) \mathrm{e}^{\mathrm{i} k x^{\prime}} \\
& -\frac{\alpha}{p+k} G(L, L, p) \mathrm{e}^{\mathrm{i} p(x-L)} \mathrm{e}^{\mathrm{i} k L}-\frac{\alpha}{p-k} G(0, L, p) \mathrm{e}^{\mathrm{i} p(x-L)} \\
& -\frac{\mathrm{i} \alpha}{p^{2}-k^{2}} \mathrm{e}^{\mathrm{i} p(x-L)} \mathrm{e}^{\mathrm{i} k L}+\frac{\mathrm{i} \alpha}{p^{2}-k^{2}} \mathrm{e}^{\mathrm{i} k x}
\end{aligned}
$$


For comparison, only the last term in the r.h.s. of (8), the last three ones in (9) and the third one in (10) appear in the shutter problem.

The crucial advantage of the method is that almost all the Green functions involved in the equations above can be expanded as a sum of terms which are simple poles in $p_{n}$.

\section{Analytical structure of the p-dependent solution}

General theorems [4] and the explicit analytical derivation (Appendix B) of $G\left(x, x^{\prime}, p\right)$ show that $\left|G\left(x, x^{\prime}, p\right)\right| \sim 1 /|p| \rightarrow 0$ as $|p| \rightarrow \infty$ in the complex plane for almost any $x \in[0, L]$ and $x^{\prime} \in[0, L]$. Then the Mittag-Leffler theorem tells that

$$
G\left(x, x^{\prime}, p\right)=\sum_{n} \frac{C_{n}\left(x, x^{\prime}\right)}{p-p_{n}}
$$

The exception is for $G(0,0, p)$ and $G(L, L, p)$, the modulus of which grows as $|p|$ for $|p| \rightarrow \infty$ in the lower half complex plane $p$ and requires some special care (Appendix $\mathrm{C})$.

The residues in the r.h.s of (11) can be easily computed [3] and one finds $C_{n}\left(x, x^{\prime}\right)=u_{n}(x) u_{n}\left(x^{\prime}\right) / N_{n}$, where the functions $u_{n}(x)$ belong to the denumerable set of the resonant solutions satisfying $L_{x} u_{n}(x)=0$ with RBCs

$$
\left.\partial_{x} u_{n}(x)\right|_{x=0}=-\mathrm{i} p_{n} u_{n}(0),\left.\partial_{x} u_{n}(x)\right|_{x=L}=\mathrm{i} p_{n} u_{n}(L)
$$

and $N_{n}$ are suitable normalization factors (Appendix A).

The inverse Laplace transform

$\psi(x, t)=\frac{1}{2 \pi \mathrm{i}} \int_{c-\mathrm{i} \infty}^{c+\mathrm{i} \infty} \mathrm{d} s \mathrm{e}^{s t} \bar{\psi}(x, p(s))=\frac{1}{2 \pi m} \int_{-\infty}^{+\infty} \mathrm{d} p p \mathrm{e}^{-\mathrm{i} \frac{p^{2}}{2 m} t} \bar{\psi}(x, p)$,

written as an integral over the real momentum variable $p$ (Appendix D), leads one to consider the pole expansion of $p \bar{\psi}(x, p)$ in the integrand, which can be worked out for each region $I, I I$ and $I I I$ (Appendix E):

$$
\begin{aligned}
p \bar{\psi}_{I}(x, p)= & \mathrm{i} \alpha \mathrm{e}^{-\mathrm{i} p x} \sum_{n} \frac{p}{p-p_{n}} \frac{u_{n}(0)}{N_{n}} \int_{0}^{L} \mathrm{~d} x^{\prime} u_{n}\left(x^{\prime}\right) \mathrm{e}^{\mathrm{i} k x^{\prime}} \\
& +\alpha \frac{k}{p+k} G(L, 0,-k) \mathrm{e}^{-\mathrm{i} p x} \mathrm{e}^{\mathrm{i} k L}-\alpha \sum_{n} \frac{p_{n}}{p-p_{n}} \frac{1}{k+p_{n}} \frac{u_{n}(L) u_{n}(0)}{N_{n}} \mathrm{e}^{-\mathrm{i} p x} \mathrm{e}^{\mathrm{i} k L} \\
& +\alpha \frac{p}{k} G(0,0,0) \mathrm{e}^{-\mathrm{i} p x}-\alpha \frac{1}{k} \frac{p^{2}}{p-k} G(0,0, k) \mathrm{e}^{-\mathrm{i} p x} \\
& +\alpha \sum_{n} \frac{p^{2}}{p-p_{n}} \frac{1}{p_{n}} \frac{1}{k-p_{n}} \frac{u_{n}^{2}(0)}{N_{n}} \mathrm{e}^{-\mathrm{i} p x}+\frac{\mathrm{i} \alpha}{2}\left(\frac{1}{p-k}+\frac{1}{p+k}\right)\left(\mathrm{e}^{\mathrm{i} k x}-\mathrm{e}^{-\mathrm{i} p x}\right) \\
p \bar{\psi}_{I I}(x, p)= & \mathrm{i} \alpha \sum_{n} \frac{p}{p-p_{n}} \frac{u_{n}(x)}{N_{n}} \int_{0}^{L} \mathrm{~d} x^{\prime} u_{n}\left(x^{\prime}\right) \mathrm{e}^{\mathrm{i} k x^{\prime}} \\
& +\alpha \frac{k}{p+k} G(L, x,-k) \mathrm{e}^{\mathrm{i} k L}-\alpha \mathrm{e}^{\mathrm{i} k L} \sum_{n} \frac{p_{n}}{p-p_{n}} \frac{1}{k+p_{n}} \frac{u_{n}(L) u_{n}(x)}{N_{n}} \\
& -\alpha \frac{k}{p-k} G(0, x, k)+\alpha \sum_{n} \frac{p_{n}}{p-p_{n}} \frac{1}{k-p_{n}} \frac{u_{n}(0) u_{n}(x)}{N_{n}}
\end{aligned}
$$




$$
\begin{aligned}
p \bar{\psi}_{I I I}(x, p)= & \mathrm{i} \alpha \mathrm{e}^{\mathrm{i} p(x-L)} \sum_{n} \frac{p}{p-p_{n}} \frac{u_{n}(L)}{N_{n}} \int_{0}^{L} \mathrm{~d} x^{\prime} u_{n}\left(x^{\prime}\right) \mathrm{e}^{\mathrm{i} k x^{\prime}} \\
& -\alpha \frac{p}{k} G(L, L, 0) \mathrm{e}^{\mathrm{i} p(x-L)} \mathrm{e}^{\mathrm{i} k L}+\frac{p^{2}}{p+k} \frac{\alpha}{k} G(L, L,-k) \mathrm{e}^{\mathrm{i} p(x-L)} \mathrm{e}^{\mathrm{i} k L} \\
& -\alpha \sum_{n} \frac{p^{2}}{p-p_{n}} \frac{1}{p_{n}} \frac{1}{k+p_{n}} \frac{u_{n}^{2}(L)}{N_{n}} \mathrm{e}^{\mathrm{i} p(x-L)} \mathrm{e}^{\mathrm{i} k L} \\
& +\alpha \sum_{n} \frac{p_{n}}{p-p_{n}} \frac{1}{k-p_{n}} \frac{u_{n}(0) u_{n}(L)}{N_{n}} \mathrm{e}^{\mathrm{i} p(x-L)}+\alpha \frac{k}{k-p} G(0, L, k) \mathrm{e}^{\mathrm{i} p(x-L)} \\
& +\frac{\mathrm{i} \alpha}{2}\left(\frac{1}{p+k}+\frac{1}{p-k}\right)\left(\mathrm{e}^{\mathrm{i} k x}-\mathrm{e}^{\mathrm{i} p(x-L)} \mathrm{e}^{\mathrm{i} k L}\right)
\end{aligned}
$$

In the third row of (14) and in the fourth row of (15) one recognizes the terms of the shutter problem given in [2]. The shutter terms in the region I, not calculated in [2], are given in the third and fourth rows of (13).

\section{The time-dependent solution}

For each of the terms in the expressions above, the integrals stemming from (12) can be brought to the form of an integral representation of the error function $\operatorname{erfc}(z)$ so that their inverse Laplace transform can be carried out thoroughly (Appendix F). We obtain:

$$
\begin{aligned}
\psi_{I}(x, t)= & -\sum_{n} \frac{u_{n}(0)}{N_{n}}\left(\int_{0}^{L} \mathrm{~d} x^{\prime} u_{n}\left(x^{\prime}\right) \mathrm{e}^{\mathrm{i} k x^{\prime}}\right)\left[A_{n}^{I}\right] \\
& +\mathrm{i} k G(L, 0,-k) \mathrm{e}^{\mathrm{i} k L}\left[B_{-k}^{I}\right]-\mathrm{i} \mathrm{e}^{\mathrm{i} k L} \sum_{n} \frac{p_{n}}{k+p_{n}} \frac{u_{n}(L) u_{n}(0)}{N_{n}}\left[B_{n}^{I}\right] \\
& +\mathrm{i} G(0,0,0) \frac{1}{k}\left[S_{0}^{I}\right]-\mathrm{i} G(0,0, k) \frac{1}{k}\left[S_{k}^{I}\right]+\mathrm{i} \sum_{n} \frac{1}{p_{n}} \frac{1}{k-p_{n}} \frac{u_{n}^{2}(0)}{N_{n}}\left[S_{n}^{I}\right] \\
& -\frac{1}{2} \mathrm{e}^{\mathrm{i} k x}\left[S_{1}^{I}\right]-\frac{1}{2} \mathrm{e}^{\mathrm{i} k x}\left[S_{2}^{I}\right]+\frac{1}{2}\left[S_{3}^{I}\right]+\frac{1}{2}\left[S_{4}^{I}\right]
\end{aligned}
$$

where the factors in square brackets embody the time $(\tau=t / 2 m)$ dependence of the solution and are the result of the integrations over the momentum $p$, namely:

$$
\begin{aligned}
& A_{n}^{I}=-\mathrm{e}^{\mathrm{i} \frac{x^{2}}{4 \tau}}\left(p_{n} \mathrm{e}^{y_{-x, n}^{2}} \operatorname{erfc}\left(y_{-x, n}\right)-\frac{\mathrm{e}^{\mathrm{i} \frac{\pi}{4}}}{\sqrt{\pi \tau}}\right) \\
& B_{-k}^{I}=\mathrm{e}^{\mathrm{i} \frac{x^{2}}{4 \tau}} \mathrm{e}^{y_{-x,-k}^{2}} \operatorname{erfc}\left(y_{-x,-k}\right) \\
& B_{n}^{I}=\mathrm{e}^{\mathrm{i} \frac{x^{2}}{4 \tau}} \mathrm{e}^{y_{-x, n}^{2}} \operatorname{erfc}\left(y_{-x, n}\right) \\
& S_{0}^{I}=\frac{\mathrm{e}^{\mathrm{i} \frac{\pi}{4}}}{\sqrt{\pi \tau}} \frac{x}{2 \tau} \mathrm{e}^{\mathrm{i} \frac{x^{2}}{4 \tau}} \\
& S_{k}^{I}=-\mathrm{e}^{\mathrm{i} \frac{x^{2}}{4 \tau}}\left(k^{2} \mathrm{e}^{y_{-x, k}^{2}} \operatorname{erfc}\left(y_{-x, k}\right)-\frac{\mathrm{e}^{\mathrm{i} \frac{\pi}{4}}}{\sqrt{\pi \tau}}\left(k-\frac{x}{2 \tau}\right)\right) \\
& S_{n}^{I}=-\mathrm{e}^{\mathrm{i} \frac{x^{2}}{4 \tau}}\left(p_{n}^{2} \mathrm{e}^{y_{-x, n}^{2}} \operatorname{erfc}\left(y_{-x, n}\right)-\frac{\mathrm{e}^{\mathrm{i} \frac{\pi}{4}}}{\sqrt{\pi \tau}}\left(p_{n}-\frac{x}{2 \tau}\right)\right)
\end{aligned}
$$


Plane wave tunnelling in a square barrier

$$
\begin{aligned}
& S_{1}^{I}=-\mathrm{e}^{-\mathrm{i} \tau k^{2}} \operatorname{erfc}(\mathrm{i} \sqrt{\mathrm{i} \tau} k) \\
& S_{2}^{I}=-\mathrm{e}^{-\mathrm{i} \tau k^{2}} \operatorname{erfc}(-\mathrm{i} \sqrt{\mathrm{i} \tau} k) \\
& S_{3}^{I}=-\mathrm{e}^{\mathrm{i} \frac{x^{2}}{4 \tau}} \mathrm{e}^{y_{-x, k}^{2}} \operatorname{erfc}\left(y_{-x, k}\right) \\
& S_{4}^{I}=-\mathrm{e}^{\mathrm{i} \frac{x^{2}}{4 \tau}} \mathrm{e}^{y_{-x,-k}^{2}} \operatorname{erfc}\left(y_{-x,-k}\right)
\end{aligned}
$$

where we have introduced the variables $y_{x, q} \equiv \mathrm{e}^{-\mathrm{i} \frac{\pi}{4}}(4 \tau)^{-\frac{1}{2}}(x-2 \tau q)$ for $q=p_{n}, k,-k$.

Likewise

$$
\begin{aligned}
\psi_{I I}(x, t)= & -\sum_{n} \frac{u_{n}(x)}{N_{n}}\left(\int_{0}^{L} \mathrm{~d} x^{\prime} u_{n}\left(x^{\prime}\right) \mathrm{e}^{\mathrm{i} k x^{\prime}}\right)\left[A_{n}^{I I}\right] \\
& +\mathrm{i} k G(L, x,-k) \mathrm{e}^{\mathrm{i} k L}\left[B_{-k}^{I I}\right]-\mathrm{i} \mathrm{e}^{\mathrm{i} k L} \sum_{n} \frac{p_{n}}{k+p_{n}} \frac{u_{n}(L) u_{n}(x)}{N_{n}}\left[B_{n}^{I I}\right] \\
& -\mathrm{i} k G(0, x, k)\left[S_{k}^{I I}\right]+\mathrm{i} \sum_{n} \frac{p_{n}}{k-p_{n}} \frac{u_{n}(0) u_{n}(x)}{N_{n}}\left[S_{n}^{I I}\right]
\end{aligned}
$$

where

$$
\begin{aligned}
& A_{n}^{I I}=-p_{n} \mathrm{e}^{-\mathrm{i} \tau p_{n}^{2}} \\
& B_{-k}^{I I}=-\mathrm{e}^{-\mathrm{i} \tau k^{2}} \operatorname{erfc}(-\mathrm{i} \sqrt{\mathrm{i} \tau} k) \\
& B_{n}^{I I}=-\mathrm{e}^{-\mathrm{i} \tau p_{n}^{2}} \operatorname{erfc}\left(\mathrm{i} \sqrt{\mathrm{i} \tau} p_{n}\right) \\
& S_{k}^{I I}=-\mathrm{e}^{-\mathrm{i} \tau k^{2}} \operatorname{erfc}(\mathrm{i} \sqrt{\mathrm{i} \tau} k) \\
& S_{n}^{I I}=-\mathrm{e}^{-\mathrm{i} \tau p_{n}^{2}} \operatorname{erfc}\left(\mathrm{i} \sqrt{\mathrm{i} \tau} p_{n}\right)
\end{aligned}
$$

Finally

$$
\begin{aligned}
\psi_{I I I}(x, t)= & -\sum_{n} \frac{u_{n}(L)}{N_{n}}\left(\int_{0}^{L} \mathrm{~d} x^{\prime} u_{n}\left(x^{\prime}\right) \mathrm{e}^{\mathrm{i} k x^{\prime}}\right)\left[A_{n}^{I I I}\right] \\
& -\mathrm{i} G(L, L, 0) \frac{\mathrm{e}^{\mathrm{i} k L}}{k}\left[B_{0}^{I I I}\right]+\mathrm{i} G(L, L,-k) \frac{\mathrm{e}^{\mathrm{i} k L}}{k}\left[B_{-k}^{I I I}\right] \\
& -\mathrm{i} \mathrm{e}^{\mathrm{i} k L} \sum_{n} \frac{1}{p_{n}} \frac{1}{k+p_{n}} \frac{u_{n}^{2}(L)}{N_{n}}\left[B_{n}^{I I I}\right] \\
& -\mathrm{i} k G(0, L, k)\left[S^{I I I}\right]+\mathrm{i} \sum_{n} \frac{p_{n}}{k-p_{n}} \frac{u_{n}(0) u_{n}(L)}{N_{n}}\left[S_{n}^{I I I}\right] \\
& -\frac{1}{2} \mathrm{e}^{\mathrm{i} k x}\left[C_{1}^{I I I}\right]-\frac{1}{2} \mathrm{e}^{\mathrm{i} k x}\left[C_{2}^{I I I}\right]+\frac{1}{2} \mathrm{e}^{\mathrm{i} k L}\left[C_{3}^{I I I}\right]+\frac{1}{2} \mathrm{e}^{\mathrm{i} k L}\left[C_{4}^{I I I}\right]
\end{aligned}
$$

where

$$
\begin{aligned}
& A_{n}^{I I I}=-\mathrm{e}^{\mathrm{i} \frac{(x-L)^{2}}{4 \tau}}\left(p_{n} \mathrm{e}^{y_{x-L, n}^{2}} \operatorname{erfc}\left(y_{x-L, n}\right)+\frac{\mathrm{e}^{\mathrm{i} \frac{\pi}{4}}}{\sqrt{\pi \tau}}\right) \\
& B_{0}^{I I I}=-\frac{\mathrm{e}^{\mathrm{i} \frac{\pi}{4}}}{\sqrt{\pi \tau}} \frac{x-L}{2 \tau} \mathrm{e}^{\mathrm{i} \frac{(x-L)^{2}}{4 \tau}} \\
& B_{-k}^{I I I}=-\mathrm{e}^{\mathrm{i} \frac{(x-L)^{2}}{4 \tau}}\left(k^{2} \mathrm{e}^{y_{x-L,-k}^{2}} \operatorname{erfc}\left(y_{x-L,-k}\right)+\frac{\mathrm{e}^{\mathrm{i} \frac{\pi}{4}}}{\sqrt{\pi \tau}}\left(-k+\frac{x-L}{2 \tau}\right)\right) \\
& B_{n}^{I I I}=-\mathrm{e}^{\mathrm{i} \frac{(x-L)^{2}}{4 \tau}}\left(p_{n}^{2} \mathrm{e}^{y_{x-L, n}^{2}} \operatorname{erfc}\left(y_{x-L, n}\right)+\frac{\mathrm{e}^{\mathrm{i} \frac{\pi}{4}}}{\sqrt{\pi \tau}}\left(p_{n}+\frac{x-L}{2 \tau}\right)\right)
\end{aligned}
$$


Plane wave tunnelling in a square barrier

$$
\begin{aligned}
& S_{k}^{I I I}=-\mathrm{e}^{\mathrm{i} \frac{(x-L)^{2}}{4 \tau}} \mathrm{e}^{y_{x-L, k}^{2}} \operatorname{erfc}\left(y_{x-L, k}\right) \\
& S_{n}^{I I I}=-\mathrm{e}^{\mathrm{i} \frac{(x-L)^{2}}{4 \tau}} \mathrm{e}^{y_{x-L, n}^{2}} \operatorname{erfc}\left(y_{x-L, n}\right) \\
& C_{1}^{I I I}=-\mathrm{e}^{-\mathrm{i} \tau k^{2}} \operatorname{erfc}(-\mathrm{i} \sqrt{\mathrm{i} \tau} k) \\
& C_{2}^{I I I}=-\mathrm{e}^{-\mathrm{i} \tau k^{2}} \operatorname{erfc}(\mathrm{i} \sqrt{\mathrm{i} \tau} k) \\
& C_{3}^{I I I}=-\mathrm{e}^{\mathrm{i} \frac{(x-L)^{2}}{4 \tau}} \mathrm{e}^{y_{x-L,-k}^{2}} \operatorname{erfc}\left(y_{x-L,-k}\right) \\
& C_{4}^{I I I}=-\mathrm{e}^{\mathrm{i} \frac{(x-L)^{2}}{4 \tau}} \mathrm{e}^{y_{x-L, k}^{2}} \operatorname{erfc}\left(y_{x-L, k}\right)
\end{aligned}
$$

In the equations (16), (18) and (20) the terms $[S]$ are the ones arising in the shutter problem. The particular values of the Green function involved are calculated in Appendix B.

\section{The short time limit}

The $t \rightarrow 0$ limit is interesting both as a check of the calculation above and for the study of the scattered wave at short times. Here we aim only to recover the initial wave function $\psi(x, 0)=\mathrm{e}^{\mathrm{i} k x}$ at $t=0$, which must happen in each of the regions $I, I I$ and III.

Careful use of the limiting values of $\operatorname{erfc}(z)$ and/or $w(z)=\mathrm{e}^{-z^{2}} \operatorname{erfc}(-\mathrm{i} z)$ for $z \rightarrow 0$ and for $z \rightarrow \infty$ in different directions of the complex $z$ plane must be made. Notice that $y_{x, q}$ tends to $\infty$ in different directions for different $q$. Also the properties of the set of resonant functions $u_{n}(x)$ as a basis of the space of solutions are crucial in region II (Appendix G). Asymptotic expressions for $\operatorname{erfc}(z)$ and $w(z)$ can be used to obtain approximations to the form of $\psi(x, t)$ in each region for small values of $t$, a task that will be faced elsewhere.

In region $I$, the factor $A_{n}^{I}$ tends to $-2 \mathrm{i} \delta(x)$ and also $S_{0}^{I}, S_{k}^{I}$ and $S_{n}^{I}$ tend to a distribution concentrated in $x=0$. The factors $B_{-k}^{I}, B_{n}^{I}, S_{3}^{I}$ and $S_{4}^{I}$ vanish exactly in this limit and both $S_{1}^{I}$ and $S_{2}^{I}$ tend to -1 . Therefore, as required for $x<0$,

$$
\lim _{t \rightarrow 0} \psi_{I}(x, t)=\mathrm{e}^{\mathrm{i} k x}
$$

In region $I I$, the factors $A_{n}^{I I}, B_{-k}^{I I}, B_{n}^{I I}, S_{k}^{I I}$ and $S_{n}^{I I}$ tend to the value -1 so that the $[A]$ term in $(18)$ gives

$$
\int_{0}^{L} \mathrm{~d} x^{\prime} \sum_{n} p_{n} \frac{u_{n}(x) u_{n}\left(x^{\prime}\right)}{N_{n}} \mathrm{e}^{\mathrm{i} k x^{\prime}}=\mathrm{e}^{\mathrm{i} k x}
$$

The $[B]$ terms yield

$$
\begin{aligned}
\mathrm{i} k G(L, x,-k) \mathrm{e}^{\mathrm{i} k L}- & \mathrm{ie} \mathrm{i}^{\mathrm{i} k L} \sum_{n} \frac{p_{n}}{k+p_{n}} \frac{u_{n}(L) u_{n}(x)}{N_{n}} \\
& =\mathrm{i} k \mathrm{e}^{\mathrm{i} k L} \sum_{n} \frac{1}{-k-p_{n}} \frac{u_{n}(L) u_{n}(x)}{N_{n}}-\mathrm{ie}^{\mathrm{i} k L} \sum_{n} \frac{p_{n}}{k+p_{n}} \frac{u_{n}(L) u_{n}(x)}{N_{n}} \\
& =-\mathrm{ie}^{\mathrm{i} k L} \sum_{n} \frac{u_{n}(L) u_{n}(x)}{N_{n}}=0
\end{aligned}
$$


and likewise for the $[S]$ terms:

$$
\begin{aligned}
\mathrm{i} k G(0, x, k)-\mathrm{i} \sum_{n} & \frac{p_{n}}{k-p_{n}} \frac{u_{n}(0) u_{n}(x)}{N_{n}} \\
& =\mathrm{i} k \sum_{n} \frac{1}{k-p_{n}} \frac{u_{n}(0) u_{n}(x)}{N_{n}}-\mathrm{i} \sum_{n} \frac{p_{n}}{k-p_{n}} \frac{u_{n}(0) u_{n}(x)}{N_{n}} \\
& =\mathrm{i} \sum_{n} \frac{u_{n}(0) u_{n}(x)}{N_{n}}=0
\end{aligned}
$$

In region $I I I$, the factor $A_{n}^{I I I}$ tends to $-2 \mathrm{i} \delta(x-L)$ and also $B_{0}^{I I I}, B_{-k}^{I I I}$ and $B_{n}^{I I I}$ approach distributions concentrated in $x=L$. Thus they vanish for $x>L$. The factors $S^{I I I}, S_{n}^{I I I}, C_{3}^{I I I}$ and $C_{4}^{I I I}$ vanish exactly in this limit, whereas both $C_{1}^{I I I}$ and $C_{2}^{I I I}$ tend to -1 . Therefore the final result is that, for $x>L$, also

$$
\lim _{t \rightarrow 0} \psi_{I I I}(x, t)=\mathrm{e}^{\mathrm{i} k x}
$$

We have quoted singularities at the points $x=0$ and $x=L$ respectively in the regions $I$ and $I I I$, but these points must be excluded from the domain of respectively $\psi_{I}(x, t)$ and $\psi_{I I I}(x, t)$, as argued in Appendix $\mathrm{F}$.

\section{The large time limit}

For $\tau \rightarrow \infty$ we see that also $y_{x, q} \rightarrow \infty$ as before, but in yet different directions of the complex plane for the different $q$.

In region I, the factors $A_{n}^{I}, B_{-k}^{I}, B_{n}^{I}, S_{0}^{I}, S_{n}^{I}, S_{2}^{I}$ and $S_{4}^{I}$ vanish, whereas

$$
\begin{aligned}
& S_{k}^{I} \rightarrow-2 k^{2} \mathrm{e}^{-\mathrm{i} E_{k} t} \mathrm{e}^{-\mathrm{i} k x} \\
& S_{1}^{I} \rightarrow-2 \mathrm{e}^{-\mathrm{i} E_{k} t} \\
& S_{3}^{I} \rightarrow-2 \mathrm{e}^{-\mathrm{i} E_{k} t} \mathrm{e}^{-\mathrm{i} k x}
\end{aligned}
$$

Using equation (B.4), some of the $\mathrm{e}^{-\mathrm{i} k x}$ terms cancel out in (16) and one is left with

$$
\begin{aligned}
\lim _{t \rightarrow \infty} \psi_{I}(x, t) & =\mathrm{e}^{-\mathrm{i} E_{k} t}\left(\mathrm{e}^{\mathrm{i} k x}+R(k) \mathrm{e}^{-\mathrm{i} k x}\right) \\
& =\mathrm{e}^{-\mathrm{i} E_{k} t} \phi_{r}^{i n}(x)
\end{aligned}
$$

that is, the asymptotic scattering in solution, where $E_{k}=k^{2} / 2 m$ (see Appendix $\mathrm{H}$ ).

In region $I I$, the factors $A_{n}^{I I}, B_{-k}^{I I}, B_{n}^{I I}$ and $S_{n}^{I I}$ vanish, whereas $S_{k}^{I I} \rightarrow-2 \mathrm{e}^{-\mathrm{i} \tau k^{2}}$. Therefore, using (B.2), for $0 \leq x \leq L$ we have

$$
\begin{aligned}
\lim _{t \rightarrow \infty} \psi_{I I}(x, t) & =2 \mathrm{i} k G(0, x, k) \mathrm{e}^{-\mathrm{i} \tau k^{2}} \\
& =\mathrm{e}^{-\mathrm{i} E_{k} t} \phi_{r}^{i n}(x)
\end{aligned}
$$

Thus the infinite plane wave evolves into the same final state of the shutter initial condition also in region $I I$.

For the region $I I I$, the only non-vanishing factors are

$$
\begin{aligned}
& S_{k}^{I I I} \rightarrow-2 \mathrm{e}^{\mathrm{i} k(x-L)} \mathrm{e}^{-\mathrm{i} \tau k^{2}} \\
& C_{2}^{I I I} \rightarrow-2 \mathrm{e}^{-\mathrm{i} \tau k^{2}} \\
& C_{4}^{I I I} \rightarrow-2 \mathrm{e}^{\mathrm{i} k(x-L)} \mathrm{e}^{-\mathrm{i} \tau k^{2}}
\end{aligned}
$$


Then the terms corresponding to $C_{2}^{I I I}$ and $C_{4}^{I I I}$ in (20) cancel each other and, again using (B.2), one is left with the same asymptotic solution of the shutter

$$
\begin{aligned}
\lim _{t \rightarrow \infty} \psi_{I I I}(x, t) & =2 \mathrm{i} k G(0, L, k) \mathrm{e}^{\mathrm{i} k(x-L)} \mathrm{e}^{-\mathrm{i} \tau k^{2}} \\
& =T(k) \mathrm{e}^{\mathrm{i} k x} \mathrm{e}^{-\mathrm{i} E_{k} t} \\
& =\mathrm{e}^{-\mathrm{i} E_{k} t} \phi_{r}^{i n}(x)
\end{aligned}
$$

The finite time behaviour is thus made up of transient modes which quickly dampen out, leaving (for $k>0$ ) the scattering asymptotic solution with outgoing BC at $x=L$.

\section{Conclusions}

A solution for the time evolution of an infinite plane wave in the presence of a simple square barrier has been worked out for each of the regions of the potential and Section 4 is the main result of this work. Among other terms, this solution contains a sum of explicit analytical contributions corresponding to each of the (infinitely many) resonance poles. As in previous works in related problems [1] [2], only the location of these poles needs to be obtained by numerical methods.

In the solution obtained the shutter terms have been pinpointed. Similarly, the contributions to the time-evolved wave function coming from the segments of the initial wave function lying inside and at the right of the barrier can be identified.

As the main application of this knowledge we envisage the possibility of studying the enhancement or the suppression of the transmission of the single Fourier components of any realistic wave packet. This should provide new detailed insight on interesting phenomena like the super-luminal tunnelling [5], the breakdown of energy conservation [6] by transient interference in wave packet collisions with barriers [7] or the rising of forerunners.

The explicit solution obtained for finite time is useful for deriving approximations valid for short times, hence for the study of transient structures and forerunners. An immediate result of the work is that the large time limit yields the same stationary solution of the shutter. This again shows that the resonances contribute only to transient structures of the scattered wave.

\section{Acknowledgments}

Work supported by MEC projects BFM2002-00834 and FIS2005-05304. The authors are indebted to J. León for suggestions and helpful discussions. J. Julve acknowledges the hospitality of the Dipartimento di Fisica dell'Università di Bologna, Italy, where part of this work was done.

\section{Appendix A. Resonant solutions}

We adopt units such that $\hbar=1$. The homogeneous equation $L_{x} u(x)=0$ with the RBCs (3) has solutions only for a denumerable set of values $p_{n}$ lying in the lower complex 
plane $p$ and satisfying the condition

$$
D(p) \equiv \ominus^{2} \mathrm{e}^{\mathrm{i} p^{\prime} L}-\oplus^{2} \mathrm{e}^{-\mathrm{i} p^{\prime} L}=0,
$$

where $p^{\prime} \equiv \sqrt{p^{2}-2 m V}, \oplus \equiv p+p^{\prime}$ and $\ominus \equiv p-p^{\prime}$. One can check that if $p_{n}$ is a solution, then $-p_{n}^{*}$ is too, so that these values are in symmetrical locations with respect to the imaginary axis. We let the label $n$ take integer values $(n \neq 0)$, with the growing positive $n$ indicating the $p_{n}$ with growing real positive part and $p_{-n} \equiv-p_{n}^{*}$. One finds that $-\frac{\pi}{4}<\arg p_{n}<0$ and $\pi<\arg p_{-n}<\frac{5 \pi}{4}$.

The solutions of $L_{x} u(x)=0$ are

$$
\begin{aligned}
u_{n}(x)= & \theta(-x)\left(-2 p_{n}^{\prime}\right) \mathrm{e}^{-\mathrm{i} p_{n} x} \\
+ & \theta(x) \theta(L-x)\left(\ominus_{n} \mathrm{e}^{\mathrm{i} p_{n}^{\prime} x}-\oplus_{n} \mathrm{e}^{-\mathrm{i} p_{n}^{\prime} x}\right) \\
+ & \theta(x-L)\left(\ominus_{n} \mathrm{e}^{-\mathrm{i} \ominus_{n} L}-\oplus_{n} \mathrm{e}^{-\mathrm{i} \oplus_{n} L}\right) \mathrm{e}^{\mathrm{i} p_{n} x},
\end{aligned}
$$

up to an arbitrary multiplicative function of $p_{n}$.

The residues $C_{n}\left(x, x^{\prime}\right)=u_{n}(x) u_{n}\left(x^{\prime}\right) / N_{n}$ in (11) correspond to the following choice of (complex) "norm" 3]

$$
N_{n}=\mathrm{i}\left(u_{n}^{2}(0)+u_{n}^{2}(L)\right)+2 p_{n} \int_{0}^{L} \mathrm{~d} x u_{n}^{2}(x)
$$

which takes the value $N_{n}=-8 m V\left(p_{n} L+2 \mathrm{i}\right)$ for the solutions (A.2).

\section{Appendix B. Analytical Green function}

The analytical solution to the Green equation $L_{x} G(x, y, p)=\delta(x-y)$ for $x$ and $y$ in the interval $[0, L]$, and obeying the $\operatorname{RBCs}(7)$, can be directly constructed:

$$
\begin{aligned}
G(x, y, p)=\frac{\mathrm{i}}{2 p^{\prime}} \frac{1}{D(p)} & \left\{2 m V\left(\mathrm{e}^{\mathrm{i} p^{\prime}(L-(x+y))}+\mathrm{e}^{-\mathrm{i} p^{\prime}(L-(x+y))}\right)\right. \\
& -\oplus^{2} \mathrm{e}^{-\mathrm{i} p^{\prime} L}\left(\theta(y-x) \mathrm{e}^{\mathrm{i} p^{\prime}(y-x)}+\theta(x-y) \mathrm{e}^{\mathrm{i} p^{\prime}(x-y)}\right) \\
& \left.-\ominus^{2} \mathrm{e}^{\mathrm{i} p^{\prime} L}\left(\theta(y-x) \mathrm{e}^{-\mathrm{i} p^{\prime}(y-x)}+\theta(x-y) \mathrm{e}^{-\mathrm{i} p^{\prime}(x-y)}\right)\right\},
\end{aligned}
$$

where the symmetry $x \leftrightarrow y$ is explicit.

The limit $|p| \rightarrow \infty$ in different directions of the complex plane can be directly read out in (B.1). For any values of $x$ and $y$ in the interval $[0, L]$ the Green function vanishes as $1 / p$ or faster in any direction, with the only exception of $G(0,0, p)$ and $G(L, L, p)$, which grow as $p$ in the lower half plane, while still decreasing as $1 / p$ in the real axis.

The particular cases $G(0, x, p)$ and $G(L, x, p)$ are related to the scattering solutions $\phi^{i n}(x)$ (Appendix H). From the integral formula $\int_{0}^{L} \mathrm{~d} x\left[\phi\left(L_{x} G-\delta\left(x-x^{\prime}\right)\right)-G L_{x} \phi\right]=0$ and the use of the BCs for $\phi$ and the RBCs for $G$, one obtains [10]

$$
\begin{aligned}
& G(0, x, p)=\frac{-\mathrm{i}}{2 p} \sqrt{2 \pi} \sqrt{\frac{p}{m}} \phi_{r}^{i n}(x) \quad(0<x \leq L) \\
& G(L, x, p)=\frac{-\mathrm{i}}{2 p} \sqrt{2 \pi} \sqrt{\frac{p}{m}} \phi_{l}^{i n}(x) \quad(0 \leq x<L)
\end{aligned}
$$


If $\operatorname{Imp} \geq 0$, the limits $x=0$ in (B.2) and $x=L$ in (B.3) can be taken, namely

$$
G(0,0, p)=\phi_{r}^{i n}(0)=\frac{-\mathrm{i}}{2 p} \sqrt{2 \pi}(1+R(p))=\phi_{l}^{i n}(L)=G(L, L, p)
$$

\section{Appendix C. Pole expansion and substractions}

Whenever the Green function $G\left(x, x^{\prime}, p\right)$ vanishes as $1 / p$ in the limit $|p| \rightarrow \infty$, the Mittag-Leffler theorem applies and the validity of the pole expansion (11) is assured. This is not the case for $G(0,0, p)$ and $G(L, L, p)$, and a substraction technique must be used [4]. This is performed by using the contour integral

$$
0=\frac{1}{2 \pi \mathrm{i}} \oint_{\Gamma} \mathrm{d} z \frac{1}{z^{2}} \frac{G\left(x, x^{\prime}, z\right)}{z-p}
$$

where the contour $\Gamma \equiv\left\{C_{o}, C_{p}, C_{n}, C_{s}\right\}$ consists of small closed paths counterclock-wise encircling the poles of the integrand (namely $C_{o}$ around the double pole at $z=0, C_{p}$ around $p$ and $C_{n}$ around each $p_{n}$ ) plus a large circle $C_{s}$ clock-wise encircling all these poles. The factor $z^{-2}$ has been introduced to assure that the integrand goes as $z^{-2}$ even for $G(0,0, p)$ and $G(L, L, p)$ so that the integral over $C_{s}$ vanishes when the radius of the circle grows to $\infty$.

From (C.1) one readily obtains (see also [11])

$G\left(x, x^{\prime}, p\right)=p^{2} \sum_{n}^{\infty} \frac{1}{N_{n}} \frac{1}{p_{n}^{2}} \frac{u_{n}(x) u_{n}\left(x^{\prime}\right)}{p-p_{n}}+\left[G\left(x, x^{\prime}, 0\right)+\left.p \partial_{p} G\left(x, x^{\prime}, p\right)\right|_{p=0}\right]$

When the pole expansion (11) holds, (C.2) becomes a trivial identity, whereas for $G(0,0, p)$ and $G(L, L, p)$ a holomorphic (linearly growing with $|p|$ ) part remains.

\section{Appendix D. Inverse Laplace transform}

The inverse Laplace transform in the variable $s$ in (12), involves an integration in the complex $s$-plane along a line parallel to the imaginary axis with $\operatorname{Rec}>0$. In the $p$-plane (recall $p=\sqrt{\mathrm{i}} \sqrt{2 m s}$ ), this path translates to a hyperbola-like one with asymptotes in the positive real and imaginary axis. For $t>0$, the factor $\mathrm{e}^{-\mathrm{i} \frac{p^{2}}{2 m} t}$ assures that it can be deformed into an integration from $+\infty$ to $-\infty$ along (and slightly above) the real axis if the integrand has poles only in the lower half plane (and on the real axis). For $t<0$, the path can be closed along a quarter circle of large radius in the 1st quadrant, thus enclosing a region without poles and giving $\psi(x, t)=0$, consistently with the Laplace method and causality [4].

\section{Appendix E. Pole expansion of $p \bar{\psi}(x, p)$}

We rewrite (8) as

$\bar{\psi}_{I I}(x, p)=\mathrm{i} \alpha \int_{0}^{L} \mathrm{~d} x^{\prime} f_{1}^{I I}\left(x^{\prime}, x, p\right) \mathrm{e}^{\mathrm{i} k x^{\prime}}-\alpha \mathrm{e}^{\mathrm{i} k L} f_{2}^{I I}(x, p)-\alpha f_{3}^{I I}(x, p)$ 
where

$$
f_{1}^{I I}\left(x^{\prime}, x, p\right)=G\left(x^{\prime}, x, p\right), f_{2}^{I I}(x, p)=\frac{G(L, x, p)}{p+k} \text { and } f_{3}^{I I}(x, p)=\frac{G(0, x, p)}{p-k} .
$$

According to the asymptotic behaviour of the $f_{i}^{I I}(p) \quad(i=1,2,3)$ above we consider the contour integrals

$$
0=\frac{1}{2 \pi \mathrm{i}} \oint_{\Gamma_{i}} \mathrm{~d} z \frac{z^{n_{i}}}{z-p} f_{i}^{I I}(z)
$$

where the values $n_{1}=0$, and $n_{2}=n_{3}=1$ are assigned so that the integrand of (E.2) behaves as $z^{-2}$ for large $z$. The contours $\Gamma_{i}$ include circles around the poles in the respective integrand (namely $p, p_{n}, k$ or $-k$ ) plus the large circle $C_{s}$, as in Appendix C. For $i=1$, equation (E.2) yields $f_{1}^{I I}(p)$, which must be multiplied by $p$ later on, whereas for $i=2,3$ one directly obtains $p f_{i}^{I I}(p)$. Collecting these results and using the pole expansion of the Green function, (14) is obtained.

The derivation of (13) and (15) follows the same lines. There we define

$$
f_{1}^{I}(p)=G\left(x^{\prime}, 0, p\right), f_{2}^{I}(p)=\frac{G(0,0, p)}{p-k}, f_{3}^{I}(p)=\frac{G(L, 0, p)}{p+k},
$$

and

$$
f_{1}^{I I I}(p)=G\left(x^{\prime}, L, p\right), f_{2}^{I I I}(p)=\frac{G(L, L, p)}{p+k}, f_{3}^{I I I}(p)=\frac{G(0, L, p)}{p-k},
$$

where the powers required re $n_{1}=0, n_{2}=-1$ and $n_{3}=1$. Notice that the negative power $n_{2}=-1$, needed by the asymptotic behaviour of $G(0,0, z)$ and $G(L, L, z)$, introduces an extra pole at $z=0$ and is related to the discussion of Appendix C.

\section{Appendix F. $\operatorname{erfc}(z)$-related integrals}

In the computation of $\psi_{I}(x, t), \psi_{I I}(x, t)$ and $\psi_{I I I}(x, t)$, the following types of integrals arise:

$$
\begin{aligned}
& I_{0}(x)=\frac{1}{\mathrm{i} \pi} \int_{-\infty}^{+\infty} \mathrm{d} p \mathrm{e}^{-\mathrm{i} \tau p^{2}} \mathrm{e}^{\mathrm{i} x p}=\frac{\mathrm{e}^{-\mathrm{i} \frac{3 \pi}{4}}}{\sqrt{\pi \tau}} \mathrm{e}^{\mathrm{i} \frac{x^{2}}{4 \tau}} \\
& I_{1}(x)=\frac{1}{\mathrm{i} \pi} \int_{-\infty}^{+\infty} \mathrm{d} p p \mathrm{e}^{-\mathrm{i} \tau p^{2}} \mathrm{e}^{\mathrm{i} x p}=\frac{\mathrm{e}^{-\mathrm{i} \frac{3 \pi}{4}}}{\sqrt{\pi \tau}} \frac{x}{2 \tau} \mathrm{e}^{\mathrm{i} \frac{x^{2}}{4 \tau}} \\
& I_{0}(x, q)=\frac{1}{\mathrm{i} \pi} \int_{-\infty}^{+\infty} \mathrm{d} p \frac{1}{p-q} \mathrm{e}^{-\mathrm{i} \tau p^{2}} \mathrm{e}^{\mathrm{i} x p} \equiv-2 M(x, q, t)=-\mathrm{e}^{\mathrm{i} \frac{x^{2}}{4 \tau}} \mathrm{e}^{y_{x, q}^{2}} \operatorname{erf} c\left(y_{x, q}\right) \\
& I_{1}(x, q)=\frac{1}{\mathrm{i} \pi} \int_{-\infty}^{+\infty} \mathrm{d} p \frac{p}{p-q} \mathrm{e}^{-\mathrm{i} \tau p^{2}} \mathrm{e}^{\mathrm{i} x p}=I_{0}(x)+q I_{0}(x, q) \\
& I_{2}(x, q)=\frac{1}{\mathrm{i} \pi} \int_{-\infty}^{+\infty} \mathrm{d} p \frac{p^{2}}{p-q} \mathrm{e}^{-\mathrm{i} \tau p^{2}} \mathrm{e}^{\mathrm{i} x p}=I_{1}(x)+q I_{0}(x)+q^{2} I_{0}(x, q)
\end{aligned}
$$

where

$$
M(x, q, t) \equiv \frac{-1}{2 \pi \mathrm{i}} \int_{-\infty}^{+\infty} \mathrm{d} p \frac{1}{p-q} \mathrm{e}^{-\mathrm{i} \frac{t}{2 m} p^{2}} \mathrm{e}^{i x p}
$$

is the Moshinsky function, often found in the literature. 
Plane wave tunnelling in a square barrier

By means of the change of variables $p \rightarrow p-\frac{x}{2 \tau}$ they can be related to the integral representation of the error function [12]

$$
w(z) \equiv \mathrm{e}^{-z^{2}} \operatorname{erfc}(-\mathrm{i} z)=-\frac{\mathrm{i}}{\pi} \int_{-\infty}^{+\infty} d u \frac{\mathrm{e}^{-u^{2}}}{u-z} \quad \operatorname{Im} \mathrm{z}>0
$$

so that

$$
M(x, q, t)=\frac{1}{2} \mathrm{e}^{\mathrm{i} \frac{m}{2 t} x^{2}} w\left(\mathrm{ie}^{-\mathrm{i} \frac{\pi}{4}} \sqrt{\frac{m}{2 t}}\left(x-\frac{t}{m} q\right)\right) .
$$

In the expressions above, is convenient the use of the variables $y_{x, q} \equiv \frac{\mathrm{e}^{-\mathrm{i} \frac{\pi}{4}}}{\sqrt{4 \tau}}(x-2 \tau q)$, where $\tau \equiv \frac{t}{2 m}$

When converting the exponent $\mathrm{i} \tau p^{2}$ into $u^{2}$, namely $u=\sqrt{\mathrm{i} \tau} p$, an integration along a line running between $\pm \sqrt{\mathrm{i}} \infty$ results. In the case $q=p_{n}$, rotating this path by an angle $\pi / 4$ towards the real axis crosses the pole $\sqrt{\mathrm{i} \tau} p_{n}$, contributing a residue. For $q= \pm k$, a small negative imaginary part is supposed (see Appendix D). The case $\operatorname{Im} z<0$ can be tackled by using the property $w\left(z^{*}\right)=w^{*}(-z)$.

The results of the integrals above, as given in (17), (19) and (21), are

$$
\begin{aligned}
& A_{n}^{I I}=I_{1}\left(0, p_{n}\right) \\
& A_{n}^{I I I}=I_{1}\left(x-L, p_{n}\right) \\
& B_{0}^{I I I}=I_{1}(x-L) \\
& A_{n}^{I}=I_{1}\left(-x, p_{n}\right) \quad B_{-k}^{I I I}=I_{2}(x-L,-k) \\
& B_{-k}^{I}=S_{4}^{I}=I_{0}(-x,-k) \quad B_{n}^{I I I}=I_{2}\left(x-L, p_{n}\right) \\
& B_{n}^{I}=S_{n}^{I}=I_{0}\left(-x, p_{n}\right) \quad S_{k}^{I I I}=I_{0}(x-L, k) \\
& S_{0}^{I}=I_{1}(-x) \quad S_{n}^{I I I}=I_{0}\left(x-L, p_{n}\right) \\
& S_{k}^{I}=I_{2}(-x, k) \quad S_{2}^{I}=B_{-k}^{I I}=C_{1}^{I I I}=I_{0}(0,-k) \\
& S_{3}^{I}=I_{0}(-x, k) \quad S_{1}^{I}=S_{k}^{I I}=C_{2}^{I I I}=I_{0}(0, k) \\
& C_{3}^{I I I}=I_{0}(x-L,-k) \\
& C_{4}^{I I I}=I_{0}(x-L, k) \\
& B_{n}^{I I}=S_{n}^{I I}=I_{0}\left(0, p_{n}\right)
\end{aligned}
$$

The computation needs some care in the limit $\tau \rightarrow 0$ when we simultaneously let $x=0$, as is the case for $A_{n}^{I I}=I_{1}\left(0, p_{n}\right)$, because the change of variables $p \rightarrow p-\frac{x}{2 \tau}$ becomes ill defined. In fact, taking first $x=0$, the naive result $A_{n}^{I I}=-p_{n} \mathrm{e}^{-\mathrm{i} \tau p_{n}^{2}} \operatorname{erfc}(\mathrm{i} \sqrt{\mathrm{i} \tau})-\frac{\mathrm{e}^{\mathrm{i} \frac{\pi}{4}}}{\sqrt{\pi \tau}}$ is singular when $\tau \rightarrow 0$. The correct result is attainable as follows: The troublesome first term in (18) describes the time evolution in region II of the initial condition $\psi_{0}(x)=\mathrm{e}^{\mathrm{i} k x}$ governed by the retarded propagator $G^{R}\left(x, x^{\prime}, t\right)$ which is the inverse Laplace transformed of the $G\left(x, x^{\prime}, p\right)$ (subject to RBCs). As such, it has the expansion

$$
G^{R}\left(x, x^{\prime}, t\right)=\sum_{n} \mathrm{e}^{-\mathrm{i} \frac{p_{n}^{2}}{2 m} t} p_{n} \frac{u_{n}(x) u_{n}\left(x^{\prime}\right)}{N_{n}},
$$

hence the result for $A_{n}^{I I}$ quoted in (19). Performing the inverse Laplace transform by integration in the variable $p$ is not convenient in this case, whereas it is more direct in the variable $s=-\mathrm{i} \frac{p^{2}}{2 m}=-\mathrm{i} E_{p}$. 
The singularities for $\tau \rightarrow 0$ reported in Section 5 have the same origin and, in this sense, the points $x=0$ and $x=L$ must be considered as belonging to the non trivial region II.

\section{Appendix G. $t \rightarrow 0, \infty$ limits of $\mathrm{e}^{y_{x, q}^{2}} \operatorname{erfc}\left(y_{x, q}\right)$}

We see that, for $t \rightarrow 0$,

$$
y_{x, q} \sim \mathrm{e}^{-\mathrm{i} \frac{\pi}{4}} \sqrt{\frac{m}{2 t}} x \rightarrow \mathrm{e}^{-\mathrm{i} \frac{\pi}{4}} \cdot \infty,
$$

regardless of $q$ being equal to $\pm k, p_{n}$ or $p_{-n}$.

For $t \rightarrow \infty$ one has

$$
y_{q} \sim \mathrm{e}^{\mathrm{i} \frac{3 \pi}{4}} \sqrt{\frac{t}{2 m}} q \rightarrow \mathrm{e}^{\mathrm{i} \phi_{q}} \cdot \infty,
$$

where $\frac{\pi}{2}<\phi_{n}<\frac{3 \pi}{4},-\frac{\pi}{4}<\phi_{-n}<0, \phi_{k}=\frac{3 \pi}{4} \quad$ and $\quad \phi_{-k}=-\frac{\pi}{4}$.

The following asymptotic formulas [12] apply:

$$
\begin{gathered}
\lim _{z \rightarrow \infty} \operatorname{erfc}(z)=0 \quad|\arg z|<\frac{\pi}{4} \\
\lim _{z \rightarrow \infty} \mathrm{e}^{z^{2}} \operatorname{erfc}(z) \sim \frac{1}{\sqrt{\pi}} \frac{1}{z}\left(1+\sum_{m=1}^{\infty} \frac{C_{m}}{z^{2 m}}\right) \rightarrow 0 \quad|\arg z|<\frac{3 \pi}{4}
\end{gathered}
$$

Only the case $\phi_{k}=\frac{3 \pi}{4}$ is out of their range, in which case the relationship

$$
\mathrm{e}^{y_{x, k}^{2}} \operatorname{erfc}\left(y_{x, k}\right)=2 \mathrm{e}^{y_{x, k}^{2}}-\mathrm{e}^{y_{x, k}^{2}} \operatorname{erfc}\left(-y_{x, k}\right),
$$

where $\left|\arg \left(-y_{x, k}\right)\right|=\frac{\pi}{4}$, is helpful.

\section{Appendix H. Scattering solutions}

The usual scattering solutions of $L_{x} \bar{\psi}(x, p)=0$ for continuous real energies $E_{p}=$ $p^{2} / 2 m>0$ may be characterized according to the homogeneous BC they obey. Contrary to the resonant solutions, which are subject to two BCs, each one of the scattering solutions solution is subject to one homogeneous $\mathrm{BC}$, alternatively at $x=0$ or at $x=L$ :

$$
\begin{aligned}
\left.\partial_{x} \phi_{r}^{\text {in }}(x)\right|_{L} & =\mathrm{i} p \phi_{r}^{i n}(L) \\
\left.\partial_{x} \phi_{l}^{\text {in }}(x)\right|_{0} & =-\mathrm{i} p \phi_{l}^{\text {in }}(0) \\
\left.\partial_{x} \phi_{r}^{\text {out }}(x)\right|_{0} & =\mathrm{i} p \phi_{r}^{\text {in }}(0) \\
\left.\partial_{x} \phi_{l}^{\text {out }}(x)\right|_{L} & =-\mathrm{i} p \phi_{l}^{\text {in }}(L),
\end{aligned}
$$

where $r(l)$ labels the right(left)-moving character of the impinging wave. Notice that the reverse notation is often found in the literature, with $r(l)$ indicating the wave coming from the right(left) of the barrier. 
These solutions, for instance

$\phi_{r}^{i n}(x, p)=\frac{1}{\sqrt{2 \pi}} \sqrt{\frac{m}{p}}\left[\theta(-x)\left(\mathrm{e}^{\mathrm{i} p x}+R \mathrm{e}^{-\mathrm{i} p x}\right)+\theta(x) \theta(L-x)\left(P \mathrm{e}^{\mathrm{i} p^{\prime} x}+Q \mathrm{e}^{-\mathrm{i} p^{\prime} x}\right)+\theta(x-L) T \mathrm{e}^{\mathrm{i} p x}\right]$,

are $\delta$-normalized in energy.

\section{References}

[1] Pérez Prieto A L, Brouard S and Muga J G 2003, J. Phys. A: Math. Gen. 362371

[2] García-Calderón G and Rubio A 1997 Phys. Rev. A 553361

[3] García-Calderón G and Peierls R 1976 Nucl. Phys. A 265443

[4] Nussenzveig H M 1972 Causality and dispersion relations (New York and London: Academic Press)

[5] León J, Julve J, Pitanga P and Urríes F J 2000 Phys. Rev. A 61062101

[6] Pérez Prieto A L, Brouard S and Muga J G 2005 Phys. Rev. A 71012703

[7] Pérez Prieto A L, Brouard S and Muga J G 2001 Phys. Rev. A 64012710

[8] García-Calderón G 1982 Lett. Nuovo Cim. 33253

[9] de la Madrid R and Gadella M 2002 Am. J. Phys. 70626

[10] García-Calderón G 1987 Sol. State Com 62441

[11] García-Calderón G 1976 Nucl. Phys. A 261130

[12] Abramowitz M and Stegun I A 1965 Handbook of Mathematical Functions (New York: Dover) p 297 